Research Article

\title{
Network Pharmacology-Based Analysis of the Effects of Corydalis decumbens (Thunb.) Pers. in Non-Small Cell Lung Cancer
}

\author{
Jun Liu $\mathbb{D}^{1}{ }^{1}$ Zhibo He, ${ }^{2}$ Shan Li, ${ }^{1}$ Wenan Huang, ${ }^{1}$ and Zhongjie Ren ${ }^{1}$ \\ ${ }^{1}$ Medical School, Jiujiang University, Jiujiang 332005, China \\ ${ }^{2}$ School of Literature and Communication, Jiujiang University, Jiujiang 332005, China \\ Correspondence should be addressed to Jun Liu; 6140115@jju.edu.cn
}

Received 3 May 2021; Revised 17 June 2021; Accepted 6 July 2021; Published 30 August 2021

Academic Editor: Jing Zhao

Copyright ( $) 2021$ Jun Liu et al. This is an open access article distributed under the Creative Commons Attribution License, which permits unrestricted use, distribution, and reproduction in any medium, provided the original work is properly cited.

Non-small cell lung cancer (NSCLC) is one of the most malignant tumors worldwide. The main treatment for NSCLC is based on Western medicine; however, the overall effect is unsatisfactory. This study aimed to investigate the potential therapeutic targets and pharmacological mechanisms of action of the traditional Chinese medicine Corydalis decumbens (Thunb.) Pers. in NSCLC based on network pharmacology and bioinformatics. The overlapping genes between Corydalis decumbens (Thunb.) Pers. and NSCLCs were screened using Venn analysis. Cytoscape 3.7.1 software was used to analyze the overlapping target protein-protein interaction (PPI) network. Gene ontology and pathway enrichment analysis using the Kyoto Encyclopedia of Genes and Genomics database were performed to exploring biological functions of the overlapping genes. The gene expression profiling interactive analysis dataset was used to analyze the correlation between hub gene expression and disease. This study revealed 38 nodes with 191 edges, which may be therapeutic targets for NSCLC. PPI network analysis showed that the most likely association was between the genes $A R$ and NCOA2, NCOA2, and RXRA and ESR1 and NCOA2. These overlapping genes were mainly enriched in the estrogen signaling pathway, calcium signaling pathway, cholinergic synapse, and PI3K-Akt signaling pathway. ESR2 mRNA levels were significantly downregulated in patients with lung adenocarcinoma (LUAD) getting worse, and KDR levels were lower in lung squamous cell carcinoma (LUSC) than those in normal tissue. PTGS2 expression was correlated with the median survival time of LUAD, and ESR1 expression was correlated with the median survival time of LUSC. The application of network pharmacology revealed the potential mechanism underlying the effects of Corydalis decumbens (Thunb.) Pers. in NSCLC treatment and provided a theoretical basis for further in-depth research in this field.

\section{Introduction}

Lung cancer is one of the most malignant tumors with high morbidity and mortality rates worldwide. It is divided into non-small cell lung cancer (NSCLC) and small cell lung cancer (SCLC) based on its different morphological characteristics. NSCLC accounts for $80 \%$ of all lung cancer cases and mainly includes lung adenocarcinoma (LUAD), lung squamous cell carcinoma (LUSC), and large cell lung cancer [1]. Recent research showed that the main treatment for NSCLC is based on Western medicine, including surgery, chemotherapy, radiotherapy, targeted therapy, and immunotherapy $[2,3]$. However, the treatment for this disease is often accompanied by unsatisfactory outcomes, such as adverse reactions, easy recurrence and metastasis, poor prognosis, and high costs [4]. Thus, actively exploring safe and effective treatment methods is necessary to prevent and treat lung cancer [5].

Traditional Chinese medicine (TCM) has a history of more than 2000 years in China and mainly includes botanical, animal, and mineral medicines. It has been widely used to prevent and treat diseases, particularly in Asia [6, 7]. Studies have shown that Chinese herbal medicines can directly inhibit malignant tumor growth and proliferation [6]. For example, botanical ingredients such as alkaloids, dietary polyphenols, and saponins are the main inhibitors of lung cancer [8-10]. Corydalis decumbens (Thunb.) Pers. is botanical medicine that is rich in alkaloids and has mainly 
focused on treating cardiovascular and cerebrovascular diseases, arthritis, and myopia [11-13]; however, its use has been scarce in tumors. The current study, using network pharmacology and bioinformatics, aimed to address the following questions: does Corydalis decumbens (Thunb.) Pers. play role in treating NSCLC? Which ingredients of Corydalis decumbens (Thunb.) Pers. have an anti-NSCLC effect? What is the molecular mechanism underlying the effect of Corydalis decumbens (Thunb.) Pers. in NSCLC treatment?

\section{Materials and Methods}

2.1. Ingredients of Corydalis decumbens (Thunb.) Pers. We searched the active ingredients of Corydalis decumbens (Thunb.) Pers. using the Traditional Chinese Medicine Systems Pharmacology Database and Analysis Platform (TCMSP, http://tcmspw.Com/tcmsp.php). The Chinese pinyin name "xiatianwu" was entered in the "herb name" column. The filter conditions were oral bioavailability $(\mathrm{OB}) \geq 30 \%$ and drug similarity $(\mathrm{DL}) \geq 0.18$.

2.2. Drug Ingredient Target Network Construction. The corresponding targets of the active ingredients were obtained from TCMSP, SymMap (https://www.symmap.org/), and the Encyclopedia of Traditional Chinese Medicine (ETCM, http://www.tcmip.cn/ETCM/index.php/Home/ Index/index.html). The reviewed human database from UniPort (http://www.Uniport.org/) was used to translate target names to gene names. Cytoscape 3.7.1 software was used to construct the drug ingredients target network.

2.3. Potential Target Genes of Corydalis decumbens (Thunb.) Pers. against Lung Cancer. We used online tools such as GeneCards (https://www.Genecards.org) to identify the symbols of genes reported in NSCLC using keyword "NSCLC." UniProt online databases were used to translate symbol names to gene names. The overlapping genes of Corydalis decumbens (Thunb.) Pers. and NSCLC were identified by drawing a Venn diagram. The overlapping genes were considered as potential Corydalis decumbens (Thunb.) Pers. genes playing a role in treating NSCLC. Cytoscape 3.7.1 software was used to construct the ingredients-potential target network.

2.4. Construction of the Overlapping Target Protein-Protein Interaction (PPI) Network. The STRING database (http:// www.string-db.org/) and Cytoscape 3.7.1 software were used to elucidate the molecular mechanism underlying the antiNSCLC effect of Corydalis decumbens (Thunb.) Pers. According to the STRING database with the species limited to "Homo sapiens" and a confidence score $>0.4$, the interaction proteins were screened. The PPI network was constructed using Cytoscape 3.7.1 for further visualization and analysis (PPI node degree, node interaction scores, and hub genes).
2.5. Functional Enrichment Analysis. The Database for Annotation, Visualization and Integrated Discovery (DAVID, https://david.ncifcrf.gov) was used for functional enrichment analysis of potential genes. "Homo sapiens" was selected as the species and "OFFICIAL GENE SYMBOL" was used for identification. Gene ontology (GO) functions and the Kyoto Encyclopedia of Genes and Genomics (KEGG) pathway enrichment analysis in the DAVID online tool were performed for exploring the biological function of the genes used in the treatment of NSCLC; $P<0.05$ was considered as the cutoff criterion. In particular, GO functions include biological process (BP), molecular function (MF), and cellular component (CC). $P<0.01$ was used as the cutoff criterion.

2.6. Correlation Analysis of Hub Gene Expression and Disease. The top 10 hub genes in the PPI network were verified using the Cytoscape 3.7.1 plugin cytoHubba. The gene expression profiling interactive analysis dataset (GEPIA, http://gepia. cancer-pku.cn/) was used to analyze the expression of hub genes, such as the differential expression analysis between NSCLC tissues and normal tissues, differential expression analysis according to pathological stages of cancer, and the expression analysis associated with overall survival (OS) of the NSCLC patients $(P<0.05)$. "MCC" was used as the calculate mode, and the "display the shortest path" as the displayed options, LUAD and LUSC, were selected as the cancer type dataset. $\log 2 \mathrm{FC}$ cutoff $=2, q$ value cutoff $=0.05$, and the other options were set as the default values.

\section{Results}

3.1. Potential Target Genes of Corydalis decumbens (Thunb.) Pers. A total of 6 ingredients and 67 targets of Corydalis decumbens (Thunb.) Pers. were screened (Table 1, Figure 1, Table S1). A total of 3475 NSCLC target genes were obtained from the GeneCards database. After the comparative analysis, the overlapping genes, which might be potential therapeutic targets for NSCLC, were also obtained. The Venn diagram showed that 32 overlapping genes of $\mathrm{Co}$ rydalis decumbens (Thunb.) Pers. participated in the regulation of NSCLC progression (Figure 2, Table S2). The generated visual PPI network of potential therapeutic targets for Corydalis decumbens (Thunb.) Pers. contained 38 nodes and 191 edges (Figure 3).

3.2. PPI Network Analysis. Based on the potential pharmacodynamics of Corydalis decumbens (Thunb.) Pers. against NSCLC, the interacting proteins were screened using the STRING online tool. We found 31 nodes (PRSS1 did not interact with any of the genes) with 90 PPI relationships in the network (Figure 4(a)). The PPI network was constructed using Cytoscape 3.7.1 software for further visualization and analysis (Figure 4(b)). The results showed 15 genes whose degree was greater than or equal to 5.8 (average score) (Table S3) and 40 edges whose combined score was greater than or equal to 0.69 (average score) (Table S4). We found possible PPIs between Corydalis decumbens (Thunb.) Pers. 
Table 1: Information of Corydalis decumbens (Thunb.) Pers. ingredients.

\begin{tabular}{lccr}
\hline Mol. ID & Name & OB (100\%) & DL \\
\hline MOL000781 & PDSP1_000624 & 33.45 & 0.69 \\
MOL000785 & Palmatine & 64.6 & 0.65 \\
MOL000787 & Fumarine & 59.26 & 0.83 \\
MOL000790 & Isocorypalmine & 35.77 & 0.59 \\
MOL000791 & Bicuculline & 69.67 & 0.88 \\
MOL000793 & C09367 & 47.54 & 0.69 \\
\hline
\end{tabular}

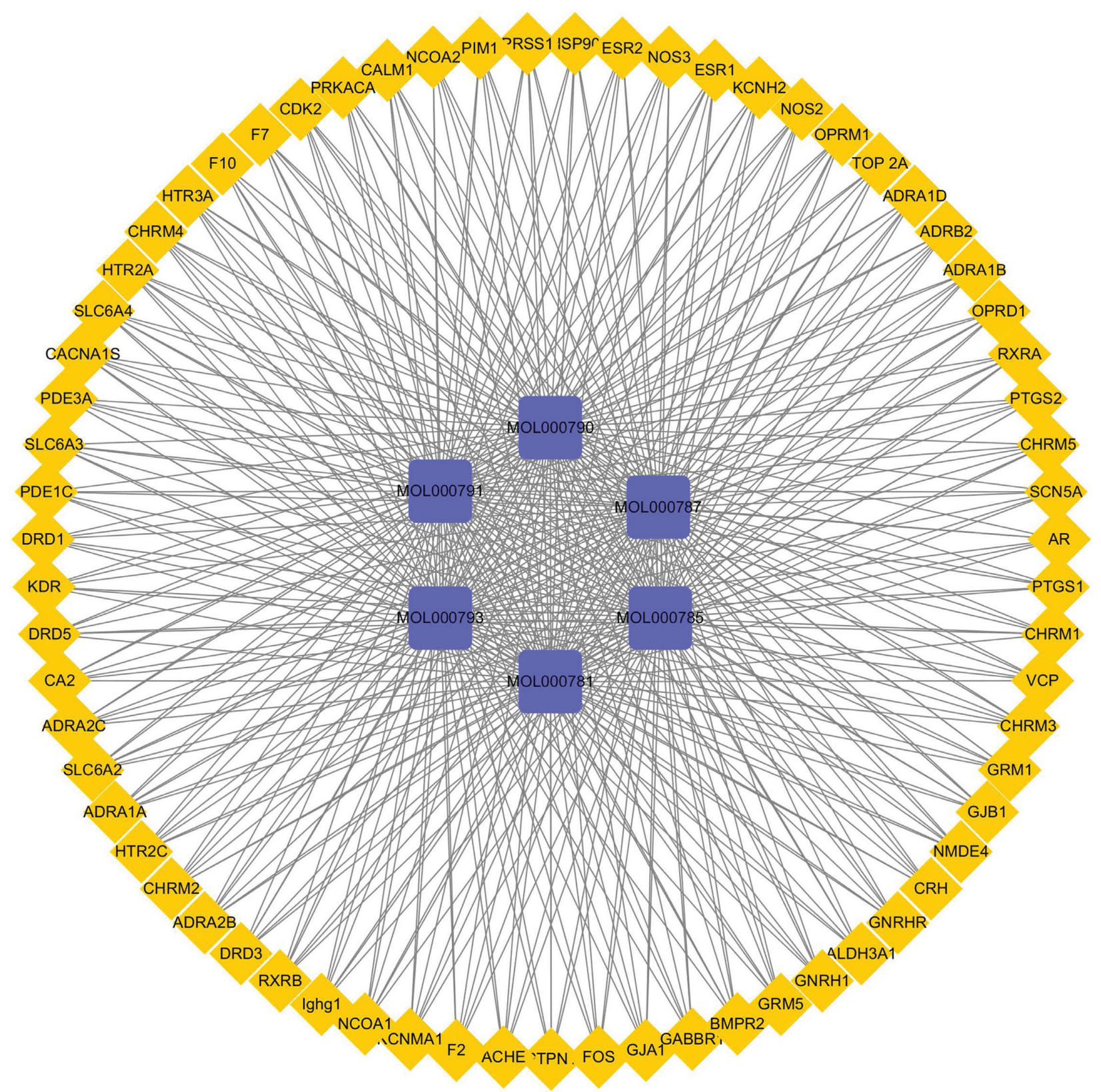

Figure 1: The ingredient-target network in the current study. The yellow diamond represents targets, and the purple rectangle represents the ingredients of Corydalis decumbens (Thunb.) Pers. The line between the two nodes represents the interaction.

and NSCLC-associated targets. The most likely association was between AR and NCOA2 (score $=0.999$ ). The remaining interactions were as follows: NCOA2 (interacts with) RXRA,
ESR1 (interacts with) NCOA2, CALM1 (interacts with) NOS2, CALM1 (interacts with) NOS3, ESR1 (interacts with) NOS3. 


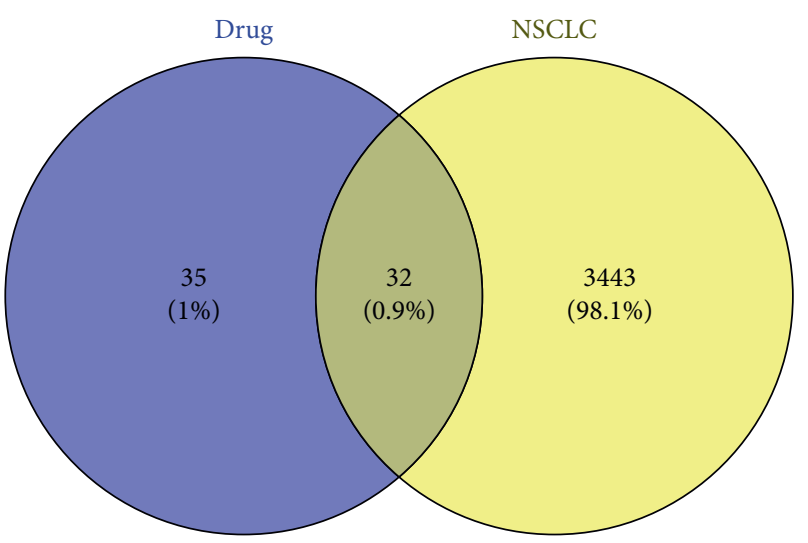

FIGURE 2: The overlapping genes in the Venn diagram.

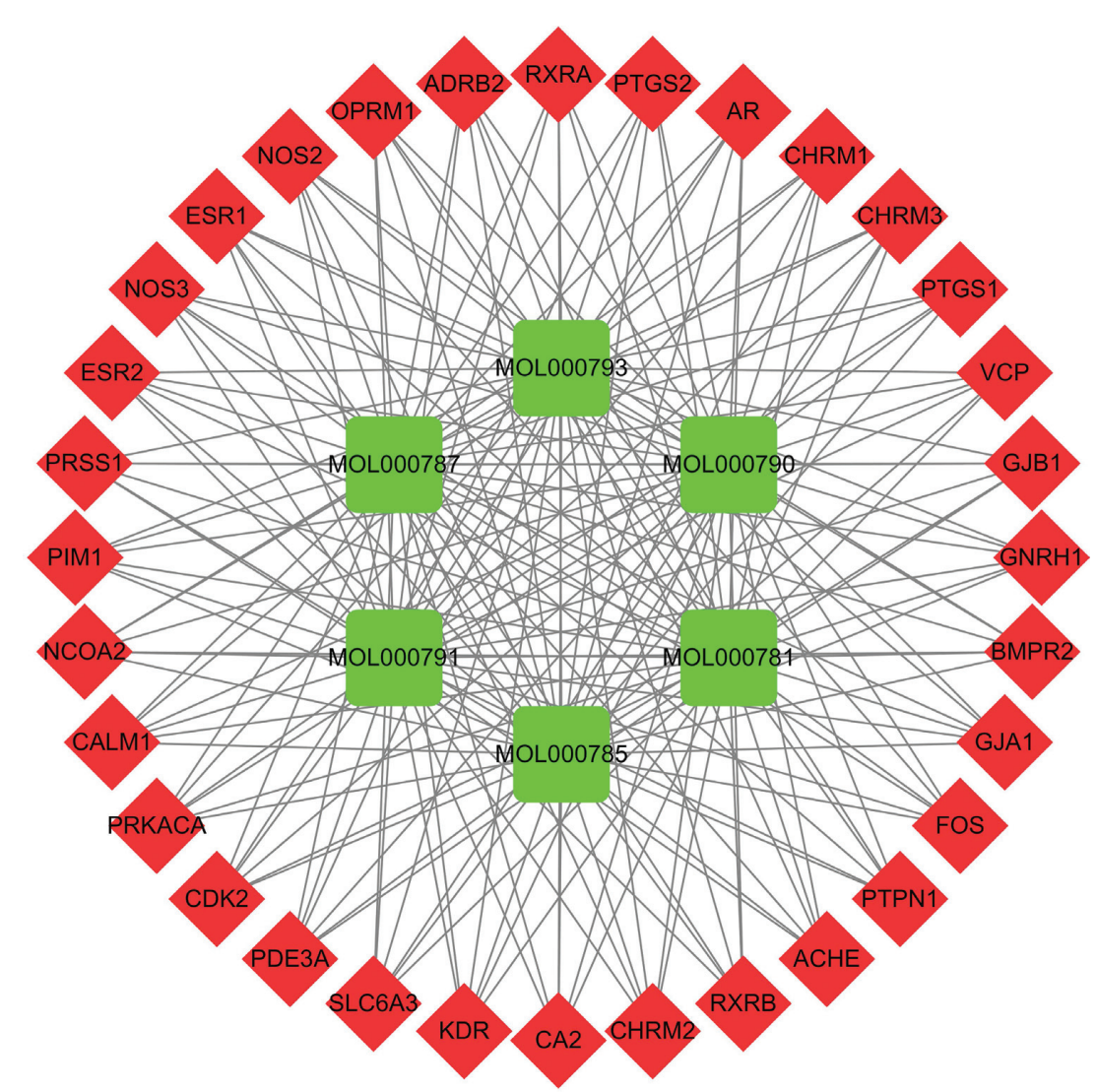

FIgUre 3: The ingredient-potential target network. The green rectangle represents the ingredients of Corydalis decumbens (Thunb.) Pers., and the red diamond represents the overlapping targets; the line between two nodes represents the interaction.

3.3. Functional Enrichment Analysis of Corydalis decumbens (Thunb.) Pers. Potential targets of Corydalis decumbens (Thunb.) Pers. were used for further GO function and pathway enrichment analyses using the DAVID online tool. The results showed that the genes could be assigned to different GO terms, which mainly involved biological processes (BP) (90 items), cell components (CC) (27 items), and molecular functions (MF) (41 items). The cutoff for statistical significance was set $P<0.01$, and the top 20 items with significant enrichment in biological processes, cell components, and molecular functions were screened, as shown in
Figure 5. For biological processes, potential therapeutic targets of Corydalis decumbens (Thunb.) Pers. were significantly enriched in "adenylate cyclase-inhibiting G-protein coupled acetylcholine receptor signaling pathway" and "phospholipase C-activating G-protein coupled acetylcholine receptor signaling pathway." For cell components, "axon terminus" and "asymmetric synapse" were mainly enriched. In addition, "RNA polymerase II transcription factor activity," ligand-activated sequence-specific DNA binding," "steroid hormone receptor activity", and "Gprotein coupled acetylcholine receptor activity" belonged to 


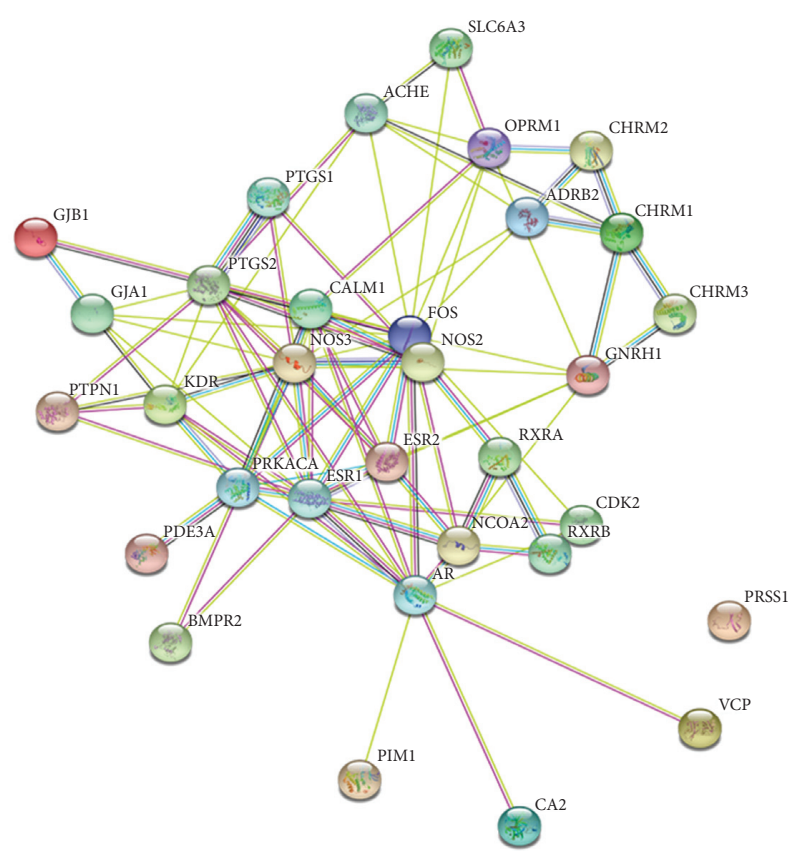

(a)

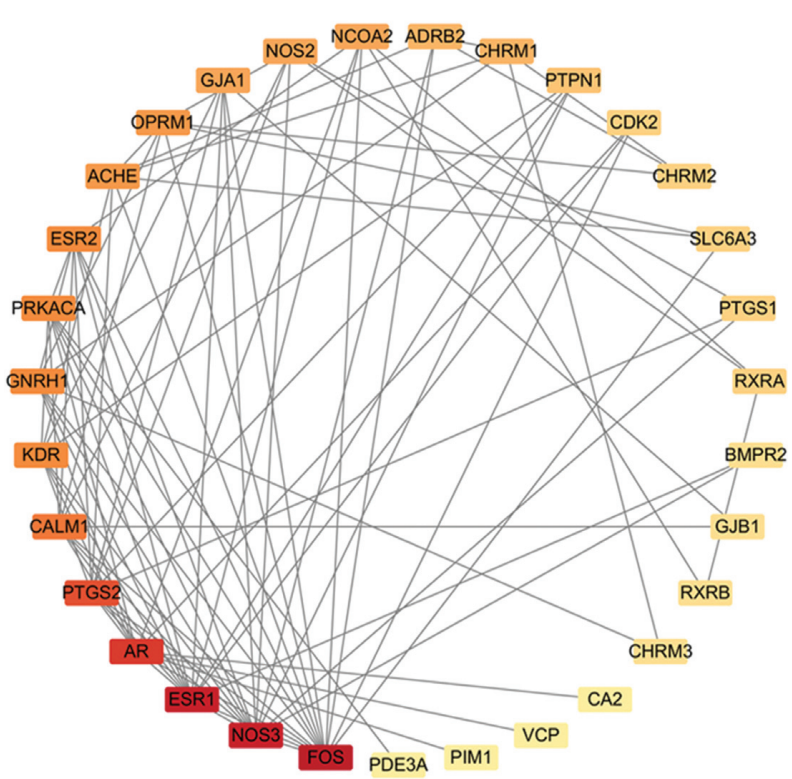

(b)

FIGURE 4: The protein-protein interaction network of overlapping genes. (a) Result from the STRING online tool. (b) Result from Cytoscape 3.7.1 software. The darker the color is, the greater the degree is.

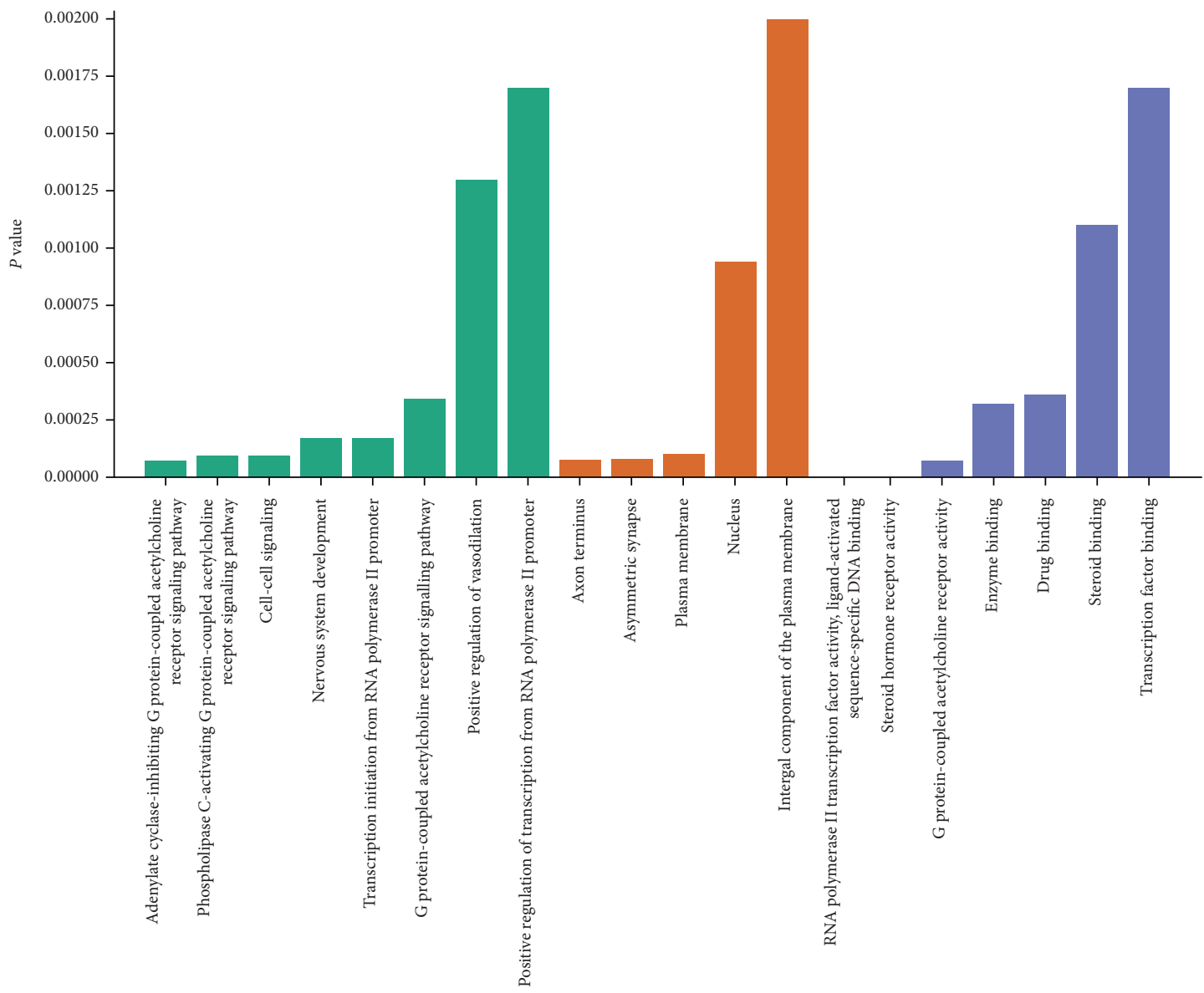




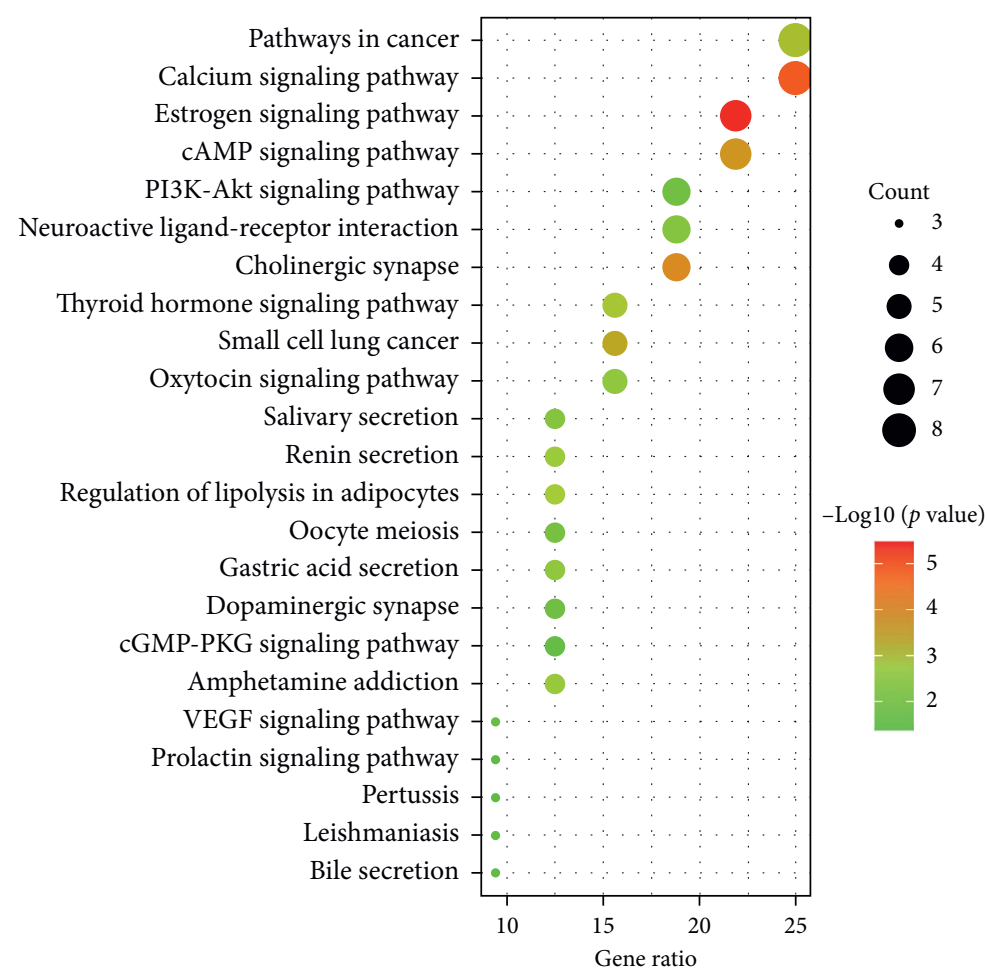

Figure 6: Pathway enrichment analysis of potential target genes of Corydalis decumbens (Thunb.) Pers. against non-small cell lung cancer using the Kyoto Encyclopedia of Genes and Genomics.

molecular functions. Additionally, the KEGG pathway enrichment analysis $(P<0.01)$ showed that these overlapping genes were mainly enriched in pathways such as the estrogen signaling pathway, calcium signaling pathway, cholinergic synapse, cAMP signaling pathway, PI3K-Akt signaling pathway, and VEGF signaling pathway (Figure 6).

3.4. Hub Genes Analysis. The hub genes of Corydalis decumbens (Thunb.) Pers. for treating NSCLC were selected based on the Cytoscape 3.7.1 plugin cytoHubba (Figure 7). Potential therapeutic targets of Corydalis decumbens (Thunb.) Pers. included CALM1, AR, ESR1, ESR2, FOS, GJA1, KDR, NOS3, OPRM1, and PRKACA. Using the GEPIA dataset, we compared the mRNA expression of hub genes among NSCLC (LUAD and LUSC) at different stages. The results showed that not all of the ESR2 mRNA expression levels were the same in different stage LUAD of patients $(P<0.05)$ (Figure 8$)$. Additionally, we compared the transcriptional levels of hub genes between NSCLC and normal samples using the GEPIA dataset. The results indicated that the expression levels of KDR were lower in LUSC than in normal tissue (Figure 9). We further explored the critical efficiency of hub genes in the survival of patients with NSCLC. GEPIA "Survival analysis" tools were used to analyze the correlation of hub genes' mRNA levels in the survival of patients with NSCLC (LUAD and LUSC). The Kaplan-Meier curve and log-rank test analyses revealed that the expression of PTGS2 was correlated with the median survival time of LUAD and the expression of ESR1 was correlated with the median survival time of LUSC. The

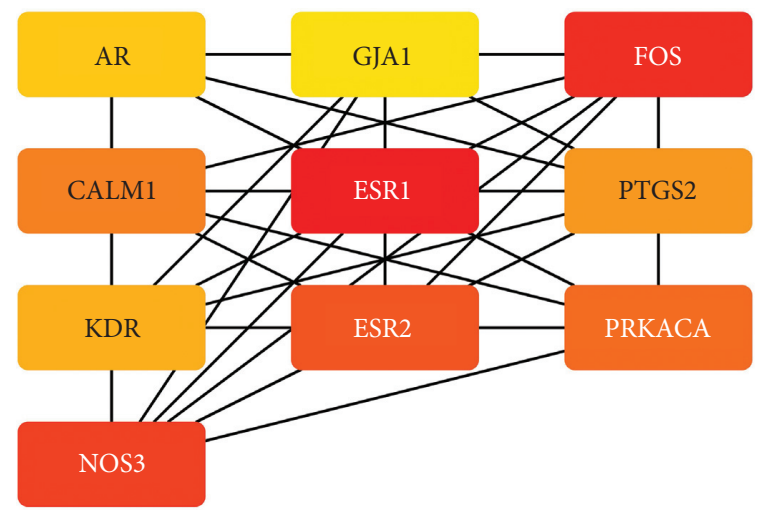

Figure 7: Top 10 hub genes of the potential target genes of $\mathrm{Co}$ rydalis decumbens (Thunb.) Pers. against non-small cell lung cancer. The darker the color is, the greater the degree is.

median survival time of the group with a high expression of PTGS2 mRNA in LUAD was lower than that of the group with low expression of PTGS2 (Figure 10(a)). The group with high expression of ESR1 in LUSC had a lower median survival time than the group with low expression of ESR1 (Figure 10(b)).

\section{Discussion}

Lung cancer is one of the most malignant cancers worldwide, and the effect of clinical treatment for this disease is not satisfactory [4]; therefore, this study explored the potential therapeutic effects of Corydalis decumbens (Thunb.) 

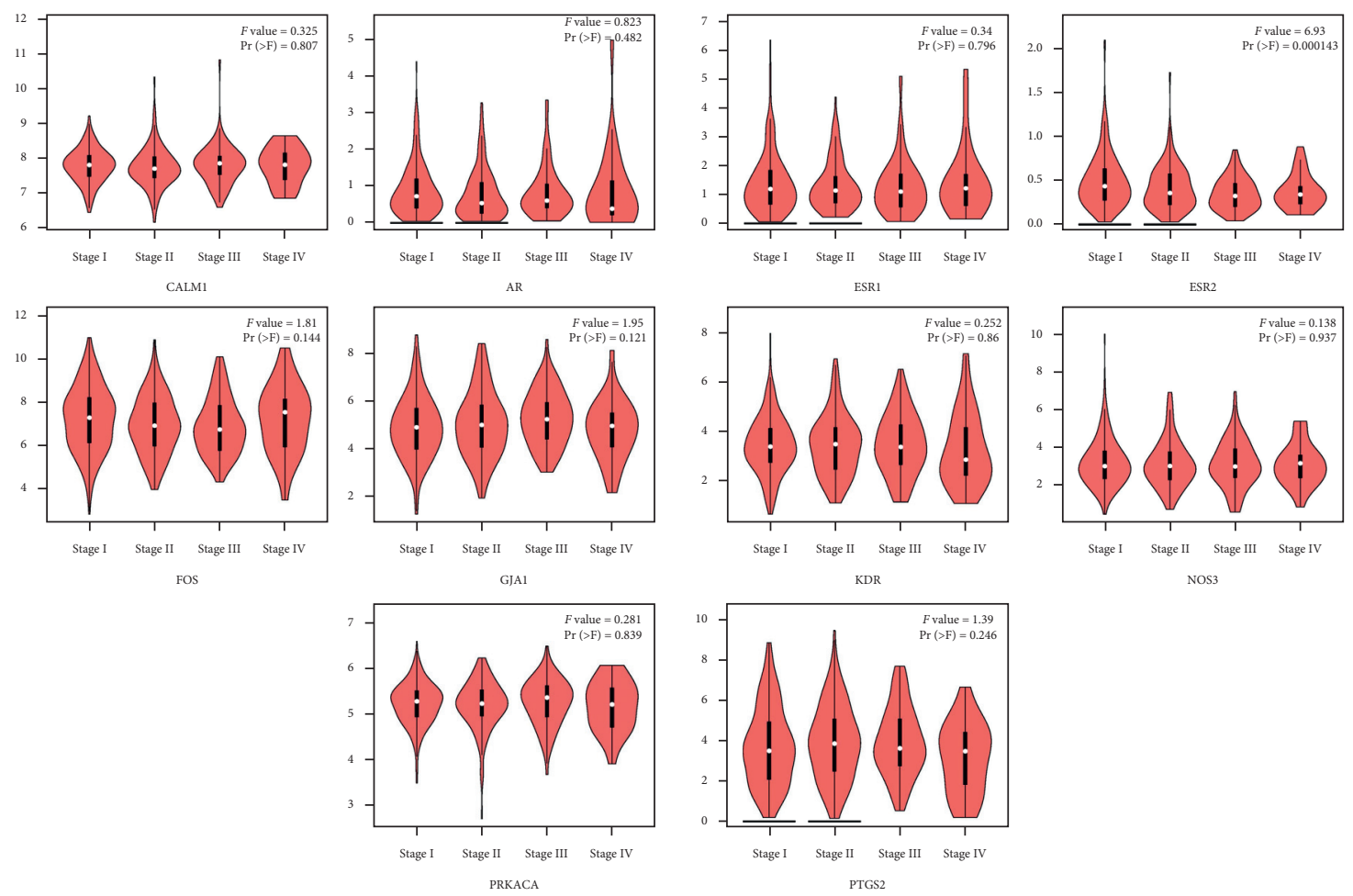

(a)
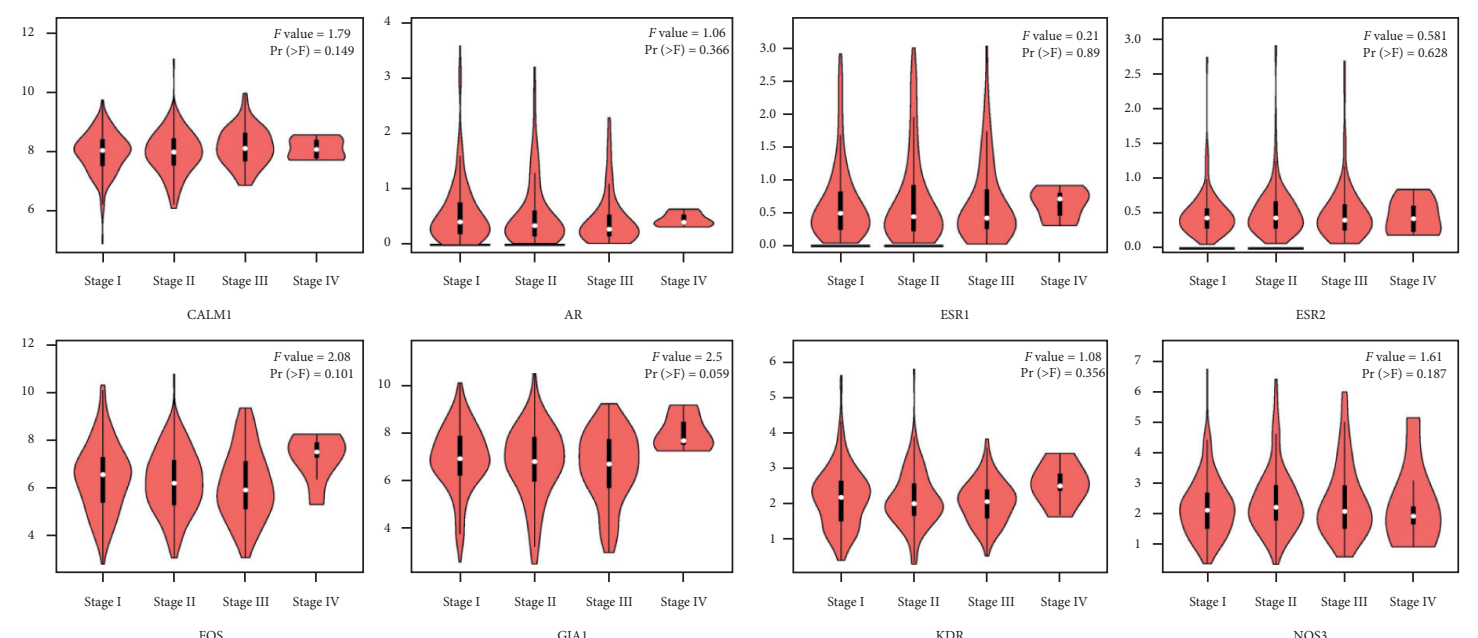

FOS

GIA1
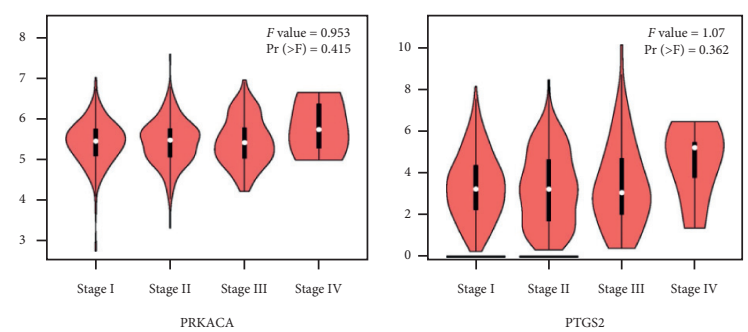

(b)

FIGURE 8: Correlation analysis among hub genes expression and tumor stage in non-small cell lung cancer patients. (a) LUAD patients. (b) LUSC patients. 

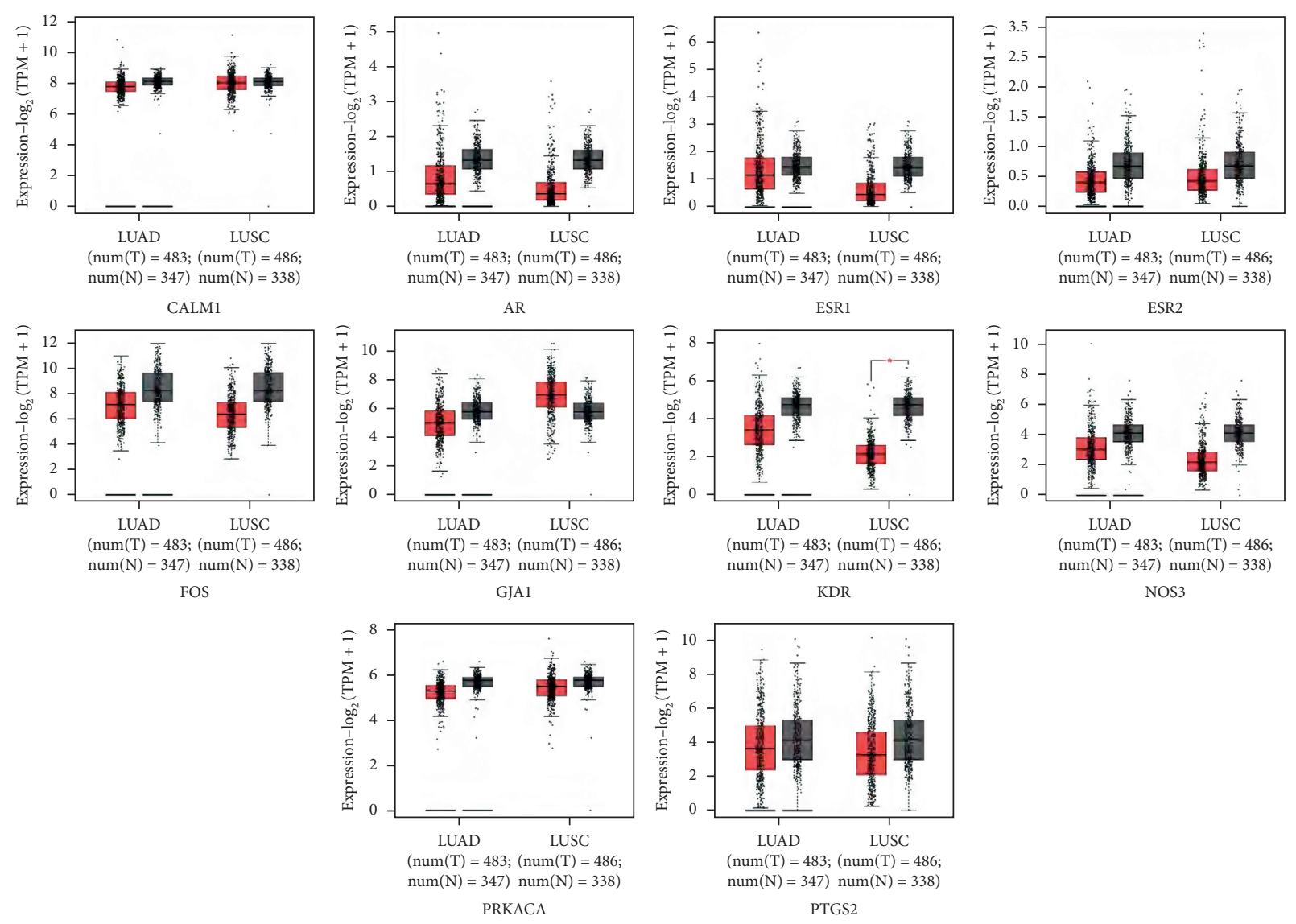

FIgURE 9: The expression of hub genes in non-small cell lung cancer. " $T$ " indicates tumor tissue, and " $N$ " indicates normal tissue.

Pers. against NSCLC based on network pharmacology analysis and bioinformatics. Network pharmacology is an interdisciplinary subject that combines systems biology and pharmacology using high-throughput sequencing, genomics, and other technologies to analyze and explore the multichannel regulation of signaling pathways [14]. In this study, we predicted a potential PPI network between NSCLC and Corydalis decumbens (Thunb.) Pers. based on network pharmacology analysis. The results showed that the main ingredients of Corydalis decumbens (Thunb.) Pers. were PDSP1_000624, palmatine, fumarine, isocorypalmine, bicuculline, and C09367. Studies have shown that alkaloids exhibit good antitumor activity. Bicuculline can effectively inhibit the migration and invasion of human hepatocellular carcinoma MHCC97-H cells which is related to the downregulation of VEGF, MMP-2, and MMP-9 gene expression and inhibition of JAK2/STAT3 signaling pathway activation [15]. Fumarine exerts antiproliferative effects by stimulating the p53 pathway in human colon cancer [16], and the apoptotic rate of HT-29 cells increased significantly after photodynamic treatment with palmatine [17].

A total of 32 overlapping genes were identified between the drug targets and NSCLC molecular targets. GO and KEGG analyses showed that they were mainly assigned to different GO terms and pathways. For example, transcription factor binding, protein binding, drug binding, estrogen signaling pathway, and calcium signaling pathway promote the occurrence and metastasis of NSCLC. Shi et al. [18] found that, in nonsmoking female patients with lung adenocarcinoma, $\mathrm{Ca}^{2+}$ is an important small-molecule signal that regulates cell function. The high expression of the $\mathrm{Ca}^{2+}$ pathway transcription factor NFATc2 is considered to be a new regulator for lung cancer to initiate cell phenotype, and high NFATc2 expression predicted poor tumor differentiation, adverse recurrence-free, and cancer-specific overall survivals in human lung cancers [19]. The increase in $\mathrm{Ca}^{2+}$ concentration in the cytoplasm of cancer cells can also induce apoptosis [20]. Increasing evidence suggests that the estrogen signaling pathway may be a therapeutic target for metastatic NSCLC [21]. Babita et al. [22] indicated that, in a preclinical model of NSCLC, estrogen promoted the growth of NSCLC and increased tumor microvessel density. In the estrogen signaling pathway, estrogen and estrogen receptors are thought to play an important role in NSCLC [23]. Hormone receptors are prognostic factors indicating metastatic tropism to the bones and comparably outcome in NSCLC early stage [24]. The PI3K-AKT signaling pathway plays a major role whole in the occurrence and development of NSCLC $[25,26]$. Therefore, it is speculated that the potentially effective ingredients of Corydalis decumbens (Thunb.) Pers. mainly act on the key factors in the above pathways to effectively treat NSCLC.

Furthermore, we analyzed the top 10 potential therapeutic targets of Corydalis decumbens (Thunb.) Pers.: 

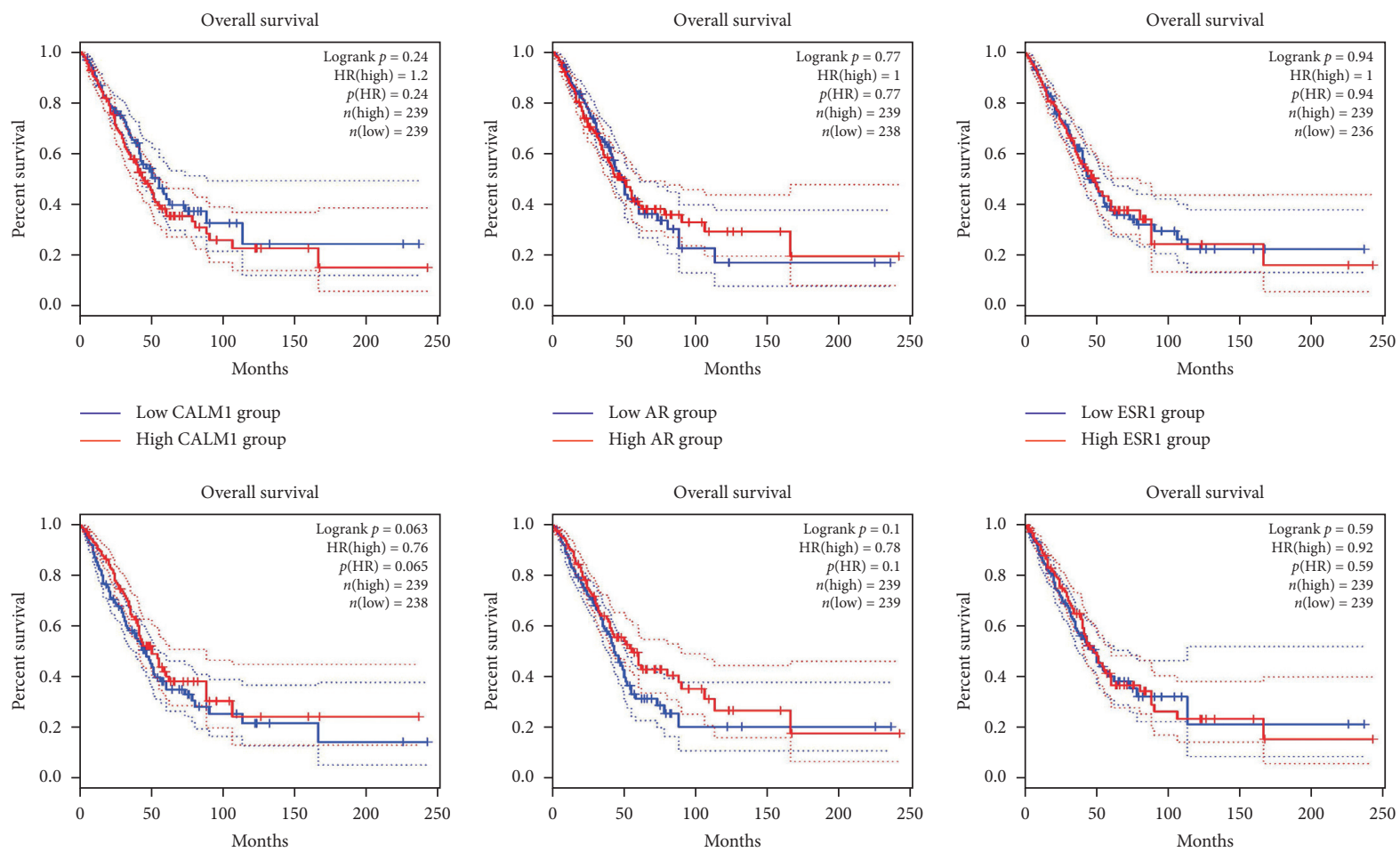

Low ESR2 group

Low FOS group

L L L NOS3 group

_ High NOS3 group
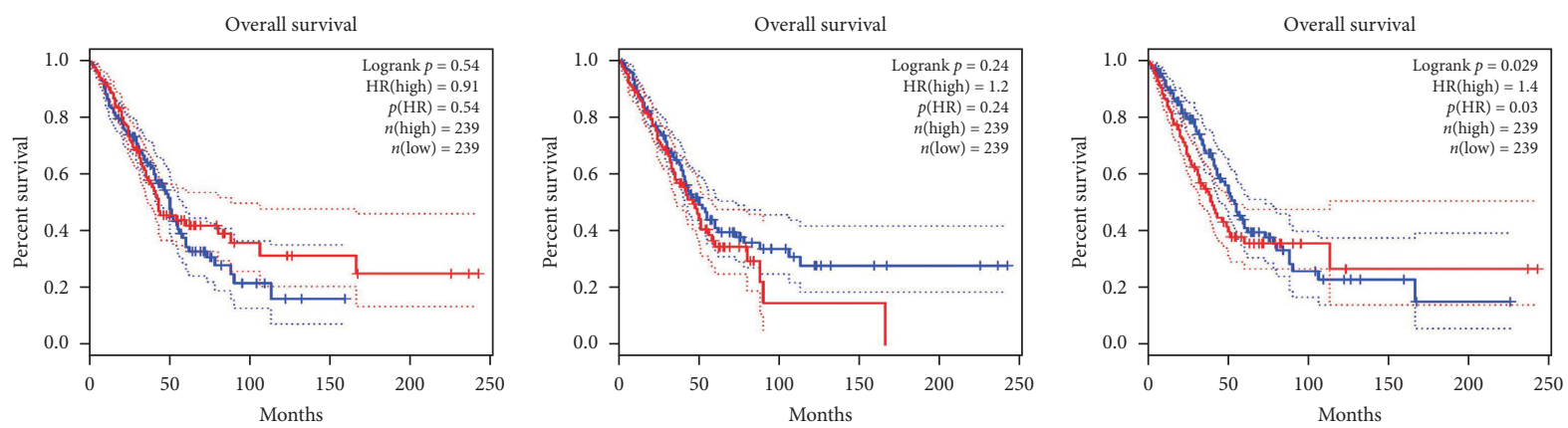

L_ Low PRKACA group
$ـ \quad$ High PRKACA group

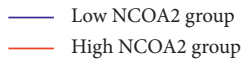

(a)

Low PTGS2 group

_ High PTGS2 group

Figure 10: Continued. 

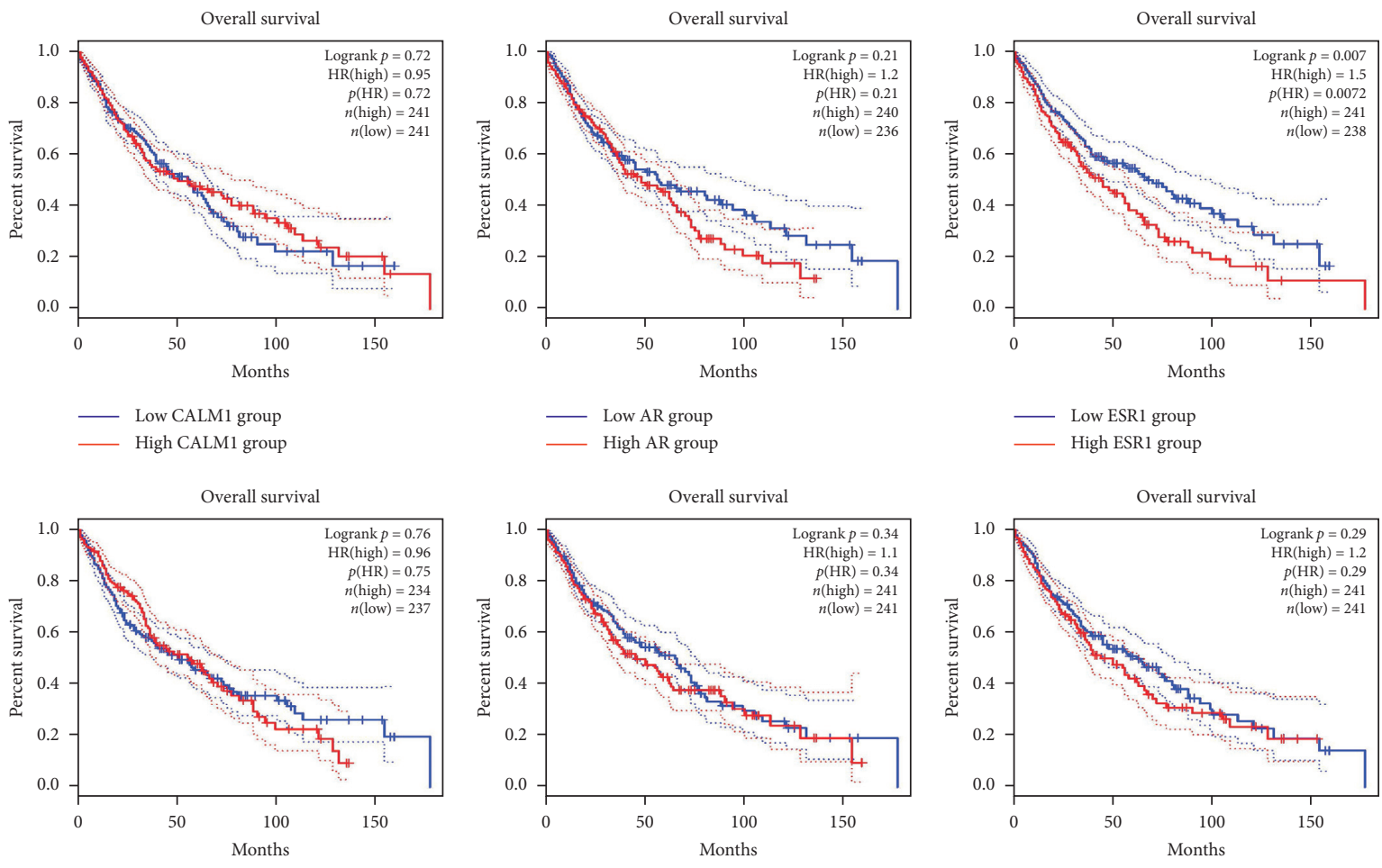

Low ESR2 group

Low FOS group

High FOS group

Low NOS3 group
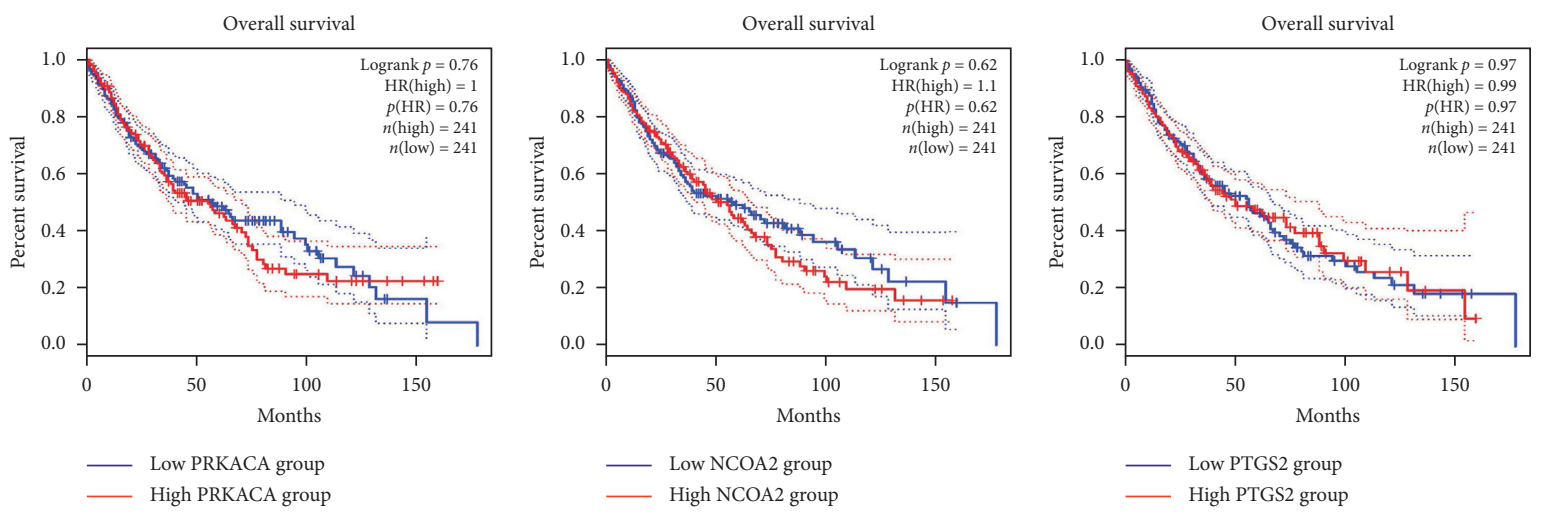

(b)

FIGURE 10: The prognostic value of mRNA of the hub factors in non-small cell lung cancer patients. (a) LUAD patients. (b) LUSC patients. The hub genes were CALM1, AR, ESR1, ESR2, FOS, NOS3, PRKACA, NCOA2, and PTGS2.

CALM1, AR, ESR1, ESR2, FOS, GJA1, KDR, NOS3, OPRM1, and PRKACA. The PPI network showed that the most likely association was between AR and NCOA2; NCOA2 and RXRA, ESR1 and NCOA2, FOS and ESR2, ESR2 and NCOA2, and FOS and ESR2. In tumor models, the adrenaline signaling pathway plays a regulatory role in tumor growth and development. Moreover, the activation of $\beta$-adrenergic receptors promotes tumor progression and development of resistance to treatment [27, 28]. Fos/Jundependent signal transduction pathways are thought to be major effects of oncogene action in NSCLC [29]; moreover, exosome-derived miR-224-5p induced NSCLC cell proliferation and metastasis by directly suppressing $A R$ [30].
Studies have found that vascular epidermal growth factor receptor 2 (VEGFR-2) plays a key role in the occurrence and development of tumors including NSCLC [31-33]. VEGFR2 is also known as the KDR in the human body. Several studies have shown that the estrogen receptor beta (ESR2) appeared in many tumors, including lung cancer, wherein ESR2 and interleukin 6 receptor (IL-6R) interacts to mediate lung cancer progression. Cell proliferation, invasion, and cell cycle were significantly increased, and cell apoptosis was markedly inhibited by the concurrent action of ESR2 and IL6 in A549 cells [34]. Chen et al. [35] showed that phosphorylated ESR1 could directly bind to the promoter of FOSL1 (FOS-like 1) in ESCs/DSCs. Moreover, silencing of 
ESR1 expression indirectly abrogated FOSL1 expression at the transcript and protein levels.

\section{Conclusions}

This study is used the network pharmacology analysis to understand the mechanism of action of Corydalis decumbens (Thunb.) Pers. and preliminarily verified the effects of Corydalis decumbens (Thunb.) Pers. in multicomponent, multitarget, and multichannel treatment of lung cancer. The application of network pharmacology revealed the potential mechanism underlying the treatment of NSCLC by $\mathrm{Co}-$ rydalis decumbens (Thunb.) Pers. and provides a theoretical basis for further in-depth research. However, this study did not consider the influence of drug dosage, drug ingredients, and drug delivery methods on the treatment results. Therefore, further experimental study is required to verify the results of this study.

\section{Data Availability}

The data used to support the findings of this study are included within the Supplementary Materials.

\section{Conflicts of Interest}

The authors declare that there are no conflicts of interest regarding the publication of this paper.

\section{Acknowledgments}

The authors would like to thank Editage (http://www. editage.cn) for English language editing. This research was supported by the Science Foundation of Department of Education of Jiangxi Province (Grant number GJJ201804) and Science Foundation of Health Commission of Jiangxi Province (Grant number 202131076).

\section{Supplementary Materials}

Table S1 is about the information of ingredients corresponding target. Table S2 is a description of overlapping genes. Table S3 is the overlapping genes degree score information. Table S4 described the combined score of interaction genes. (Supplementary Materials)

\section{References}

[1] R. L. Siegel, K. D. Miller, and A. Jemal, "Cancer statistics, 2020," CA: A Cancer Journal for Clinicians, vol. 70, no. 1, pp. 7-30, 2020.

[2] A. Upadhya, K. S. Yadav, and A. Misra, "Targeted drug therapy in non-small cell lung cancer: clinical significance and possible solutions-Part I," Expert Opinion on Drug Delivery, vol. 18, no. 1, pp. 73-102, 2021.

[3] D. Rocco, V. Gregorc, C. Lazzari, G. Palazzolo, and C. Gridelli, "New immunotherapeutic drugs in advanced non-small cell lung cancer (NSCLC): from preclinical to phase I clinical trials," Expert Opinion on Investigational Drugs, vol. 29, no. 9, pp. 1005-1023, 2020.
[4] J. Zheng, T. Xu, F. Chen, and Y. Zhang, "Correction to: MiRNA-195-5p functions as a tumor suppressor and a predictive of poor prognosis in non-small cell lung cancer by directly targeting CIAPIN1," Pathology Oncology Research: POR, vol. 26, no. 3, p. 2015, 2020.

[5] L.-Q. Wan, Y. Tan, M. Jiang, and Q. Hua, "The prognostic impact of traditional Chinese medicine monomers on tumorassociated macrophages in non-small cell lung cancer," Chinese Journal of Natural Medicines, vol. 17, no. 10, pp. 729-737, 2019.

[6] F. Qi, L. Zhao, A. Zhou et al., "The advantages of using traditional Chinese medicine as an adjunctive therapy in the whole course of cancer treatment instead of only terminal stage of cancer," Bioscience Trends, vol. 9, no. 1, pp. 16-34, 2015.

[7] Y. F. Huang, C. Bai, F. He, Y. Xie, and H. Zhou, "Review on the potential action mechanisms of Chinese medicines in treating coronavirus disease 2019 (covid-19)," Pharmacological Research, vol. 158, Article ID 104939, 2020.

[8] X. B. Wang, L. F. Li, C. Q. Cheng et al., "The role of soy food intake and EGFR/ESR2 gene SNPs in the incidence of lung cancer in non-smoking women," Journal of Public Health and Preventive Medicine, vol. 30, no. 1, pp. 23-26, 2019.

[9] M. Y. Xiang, R. L. Li, Z. W. Zhang et al., "Advances in the research of the regulation of Chinese traditional medicine monomer and its derivatives on autophagy in non-small cell lung cancer," Chinese Journal Of Lung Cancer, vol. 20, no. 3, pp. 205-212, 2017.

[10] D. Chakravarthy, A. R. Muñoz, A. Su et al., "Palmatine suppresses glutamine-mediated interaction between pancreatic cancer and stellate cells through simultaneous inhibition of survivin and COL1A1," Cancer Letters, vol. 419, pp. 103115,2018

[11] B. Tian, J. Lai, and S. M. Huang, "Effect of TAC on nerve protection in rats induced by chronic cerebral hypoperfusion," Acta Chinese Medicine and Pharmacology, vol. 48, no. 3, pp. 16-20, 2020.

[12] R. B. Pan, H. W. Zhan, J. F. Zou et al., "Intervention effect and mechanism of coydalis decumbens Pers.Alcohol extract on experimental cerebral hemorrhage in rats," Asia-Pacific Traditional Medicine, vol. 16, no. 10, pp. 29-33, 2020.

[13] X. Zhang and S. J. Yang, "Clinical observation of xiatianwu eye drops combined with raceanisodamine eye drops in treatment of pseudo myopia in children," Drugs \& Clinic, vol. 32, no. 1, pp. 116-119, 2017.

[14] J. Muhammad, A. Khan, A. Ali et al., "Network pharmacology: exploring the resources and methodologies," Current Topics in Medicinal Chemistry, vol. 18, no. 12, pp. 949-964, 2018.

[15] M. Guo, C. H. Zhang, and T. T. Wu, "Effects of dicentrine on migration and invasion of human hepatocellular carcinoma MHCC97-H cells and potential mechanisms," Chinese Traditional and Herbal Drugs, vol. 50, no. 22, pp. 5515-5520, 2019.

[16] Y. Son, Y. An, J. Jung et al., "Protopine isolated from Nandina domestica induces apoptosis and autophagy in colon cancer cells by stabilizing p53," Phytotherapy Research, vol. 33, no. 6, pp. 1689-1696, 2019.

[17] J. Wu, Q. Xiao, N. Zhang et al., "Photodynamic action of palmatine hydrochloride on colon adenocarcinoma HT-29 cells," Photodiagnosis and Photodynamic Therapy, vol. 15, pp. 53-58, 2016.

[18] K. Shi, N. Li, M. Yang, and W. Li, "Identification of key genes and pathways in female lung cancer patients who never 
smoked by a bioinformatics analysis," Journal of Cancer, vol. 10, no. 1, pp. 51-60, 2019.

[19] Z. J. Xiao, J. Liu, S. Q. Wang et al., "NFATc2 enhances tumorinitiating phenotypes through the NFATc2/SOX2/ALDH axis in lung adenocarcinoma," ELife, vol. 6, Article ID e26733, 2017.

[20] C.-L. Wang, C. Liu, L.-L. Niu, L.-R. Wang, L.-H. Hou, and X.-H. Cao, "Surfactin-induced apoptosis through ROS-ERSCa2+-ERK pathways in HepG2 cells," Cell Biochemistry and Biophysics, vol. 67, no. 3, pp. 1433-1439, 2013.

[21] M. S. Oh, J. F. Anker, and Y. K. Chae, "High gene expression of estrogen and progesterone receptors is associated with decreased t cell infiltration in patients with nsclc," Cancer Treatment and Research Communications, vol. 27, no. 3, 2021.

[22] B. Saigal, M. H. Herynk, T. Cascone et al., "Estrogen contributes to bevacizumab resistance in xenograft models of non-small cell lung cancer (NSCLC)," Cancer Research, vol. 71, no. 8, p. 3273, 2011.

[23] T. Smida, T. C. Bruno, and L. P. Stabile, "Influence of estrogen on the NSCLC microenvironment: a comprehensive picture and clinical implications," Frontiers in Oncology, vol. 10, p. 137, 2020.

[24] W. M. Brueckl, C. Eschbach, G. H. Wiest et al., "Influence of expression of estrogen (ERS-1) and progesterone (PGR) receptors on metastatic spread and outcome in non-small cell lung cancer (NSCLC)," Journal of Clinical Oncology Official Journal of the American Society of Clinical Oncology, vol. 29, no. 15, p. 7585, 2011.

[25] Y. Dan, X. Wang, S. V. Frye et al., “Abstract 1082: MerTK promotes resistance to irreversible EGFR TKIs by activation of the PI3K-AKT pathway in NSCLCs expressing wild-type EGFR," Cancer Research, vol. 77, no. 13, p. 1082, 2017.

[26] E. Golden, R. Rashwan, E. A. Woodward et al., "The oncogene AAMDC links PI3K-AKT-mTOR signaling with metabolic reprograming in estrogen receptor-positive breast cancer," Nature Communications, vol. 12, no. 1, p. 1920, 2021.

[27] G. E. Bae, H.-S. Kim, K. Y. Won, G. Y. Kim, J.-Y. Sung, and S.-J. Lim, "Lower sympathetic nervous system density and $\beta$-adrenoreceptor expression are involved in gastric cancer progression," Anticancer Research, vol. 39, no. 1, pp. 231-236, 2019.

[28] G. Boulay, N. Malaquin, I. Loison et al., "Loss of hypermethylated in cancer 1 (HIC1) in breast cancer cells contributes to stress-induced migration and invasion through $\beta-2$ adrenergic receptor (ADRB2) misregulation," Journal of Biological Chemistry, vol. 287, no. 8, pp. 5379-5389, 2012.

[29] M. Schuermann, R. Jäger, U. Salge, G. Risse-Hackl, K. Havemann, and H.-H. Heidtmann, "Control of proteinase expression by phorbol-ester-and FOS-dependent pathways in human non-small-cell lung-cancer cells," International Journal of Cancer, vol. 71, no. 2, pp. 275-283, 1997.

[30] J. Zhou, H. Wang, Q. Sun et al., "miR-224-5p-enriched exosomes promote tumorigenesis by directly targeting androgen receptor in non-small cell lung cancer," Molecular Therapy-Nucleic Acids, vol. 23, pp. 1217-1228, 2021.

[31] A. Charpidou, I. Gkiozos, M. Konstantinou et al., "Bronchial washing levels of vascular endothelial growth factor receptor2 (VEGFR2) correlate with overall survival in NSCLC patients," Cancer Letters, vol. 304, no. 2, pp. 144-153, 2011.

[32] K. Lu, M. Bhat, S. Peters, R. Mitra, T. Oberyszyn, and S. Basu, "Suppression of beta 2 adrenergic receptor actions prevent UVB mediated cutaneous squamous cell tumorigenesis through inhibition of VEGF-A induced angiogenesis," Molecular Carcinogenesis, vol. 60, no. 3, pp. 172-178, 2021.
[33] F. Song, B. Hu, J.-W. Cheng et al., "Anlotinib suppresses tumor progression via blocking the VEGFR2/PI3K/AKT cascade in intrahepatic cholangiocarcinoma," Cell Death \& Disease, vol. 11, no. 7, p. 573, 2020.

[34] H. X. Tang, Y. Q. Bai, L. C. Xiong et al., "Interaction of estrogen receptor $\beta 5$ and interleukin 6 receptor in the progression of non-small cell lung cancer," Journal of Cellular Biochemistry, vol. 120, Article ID 30216513, 2018.

[35] C. Chen, C. Li, W. Liu et al., "Estrogen-induced FOS like 1 regulates matrix metalloproteinase expression and the motility of human endometrial/decidual stromal cells," Journal of Biological Chemistry, vol. 295, no. 8, pp. 2248-2258, 2020. 